\title{
Theory of polar corrections to donor binding
}

\author{
G. D. Mahan ${ }^{1}$ and Kristian Berland ${ }^{2}$ \\ ${ }^{1}$ Department of Physics, Pennsylvania State University, University Park, Pennsylvania 16802, USA \\ ${ }^{2}$ Department of Microtechnology and Nanoscience, Chalmers Technical University, Göteborg, Sweden \\ (Received 1 July 2011; revised manuscript received 15 November 2011; published 6 December 2011)
}

\begin{abstract}
We calculate the optical-phonon correction to the binding energy of electrons to donors in cubic materials. Previous theories calculated the Rydberg energy reduced by the effective mass and the static dielectric function. They omitted an important energy term from the long-range polarization of the ionized donor, which vanishes for the neutral donor. They also omitted the donor-phonon interaction. The inclusion of these terms yields a new formula for the donor binding energy.
\end{abstract}

DOI: 10.1103/PhysRevB.84.235203

PACS number(s): 71.55.-i, 71.38.-k

\section{INTRODUCTION}

We present a calculation of the polaron correction to the binding energy $E_{D}$ of electrons to donors. This topic was treated originally by Larsen. ${ }^{1-6}$ We adopt a similar approach, but include more terms in the theory.

Prior theories are called the scaled hydrogen model (SHM). In the SHM, one takes the binding energy of hydrogen, i.e., the Rydberg energy, and scales it with the effective band mass $m^{*}$ of the electron, and the dielectric function $\varepsilon$ of the material. In SI units,

$$
E_{D}=\frac{e^{4} m^{*}}{2(4 \pi \varepsilon)^{2} \hbar^{2}}
$$

For weakly bound donors, the dielectric function is usually taken to be the zero-frequency value $\varepsilon(0)$, which includes the polar contribution from optical phonons. When the donor binding energy is larger than the optical-phonon energy, some researchers advocate using the high-frequency dielectric constant $\varepsilon(\infty)$. Neither our theory nor that of Larsen is able to derive these expressions for the SHM. We show below that our theory gives the formula for the effective dielectric constant,

$$
\frac{1}{\varepsilon}=\frac{5}{16 \varepsilon(\infty)}+\frac{11}{16 \varepsilon(0)}
$$

The fractional factors are unexpected and are the main result of our calculation. The SHM is still in regular use today. ${ }^{7-10}$

In doing this calculation, we became aware that prior theories omitted an important energy term: the polarization energy of the ionized donor. An ionized donor is viewed as a point charge in a polarizable medium. There is a polarization energy associated with this long-range potential. We calculate below the exact expression for this energy. When the electron becomes bound to the donor, the donor becomes neutral, and the long-range polarization energy vanishes. It is replaced by short-range polarization on the scale of the Bohr radius of the donor. This difference in polarization energy contributes to the binding energy of the donor. It is one reason for the fractions in Eq. (2).

We present calculations of donor binding energies for materials with the fcc (face-centered-cubic) lattice. This includes most III-V and II-VI semiconductors, and some oxides. For semiconductors with small donor binding energies, we get similar accuracy compared to the SHM. For the oxides, in particular, our theory gives much larger binding energies than does the SHM, but neither agree with experiment. Our theory may also apply to the binding of holes to acceptors. However, the degeneracy of most valence bands makes this case more complicated, and we do not consider the acceptor case.

Landau and Pekar ${ }^{1-13}$ developed the first theory of polarons for a free electron. They did a variational calculation on an electron eigenfunction, $\phi(\mathbf{r})$. They calculated the phonon displacement $\delta Q$ in response to the charge density of this eigenfunction, and then calculated the polaron energy $\propto$ $(\delta Q)^{2}$. This phonon excess energy then became part of the variational energy determining the electron eigenfunction. The Landau-Pekar theory later became recognized as the strong-coupling theory of polarons. ${ }^{14}$ Here we adopt the same procedure to calculate the response of the phonons to the electron bound to the donor. Instead of the Gaussian eigenfunction used by Landau and Pekar, we use the exponential eigenfunction typical of a donor. Otherwise our calculation follows the same procedures. Obviously, our theory is also a strong-coupling theory, which applies when $\varepsilon(\infty)$ and $\varepsilon(0)$ are very different.

\section{THEORY}

We employ an approach based on our earlier work. ${ }^{15,16}$ The Hamiltonian includes optical phonons, donors at $\mathbf{R}_{l}$ of charge $q>0$, and an electron of charge $e<0$, with effective mass $m^{*}$, which may be bound to a donor. The optical phonons have a displacement $\mathbf{Q}_{j}$, with a Szigeti charge $e^{*}$, which creates a dipole $e^{*} \mathbf{Q}_{j}$.

$$
\begin{aligned}
H= & \frac{p^{2}}{2 m}+\sum_{l} \frac{e q}{4 \pi \varepsilon(\infty)\left|\mathbf{r}-\mathbf{R}_{l}\right|}+\frac{q^{2}}{4 \pi \varepsilon(\infty)} \sum_{l>l^{\prime}} \frac{1}{\left|\mathbf{R}_{l}-\mathbf{R}_{l^{\prime}}\right|} \\
& +\sum_{j}\left[\frac{P_{j}^{2}}{2 M}+\frac{K}{2} Q_{j}^{2}\right]+V_{p d}+V_{e p}+V_{p p},
\end{aligned}
$$

where

(i) the interaction between the phonons and the donors is

$$
V_{p d}=-\frac{e^{*} q}{4 \pi \varepsilon(\infty)} \sum_{j, l} \frac{\mathbf{Q}_{j} \cdot\left(\mathbf{R}_{j}-\mathbf{R}_{l}\right)}{\left|\mathbf{R}_{j}-\mathbf{R}_{l}\right|^{3}},
$$


(ii) the interaction between the electrons and the optical phonons is

$$
V_{e p}=-\frac{e e^{*}}{4 \pi \varepsilon(\infty)} \sum_{j, l} \int d^{3} r n(r) \frac{\mathbf{Q}_{j} \cdot\left(\mathbf{R}_{j}-\mathbf{R}_{l}-\mathbf{r}\right)}{\left|\mathbf{R}_{j}-\mathbf{R}_{l}-\mathbf{r}\right|^{3}},
$$

where $n(r)$ is the charge density of an electron bound to the donor, and which reduces to the usual Fröhlich interaction for electrons in a free-energy band, ${ }^{17}$ and

(iii) the dipole-dipole interaction between the phonons is

$$
\begin{gathered}
V_{p p}=-\frac{\left(e^{*}\right)^{2}}{8 \pi \varepsilon(\infty)} \sum_{i \neq j} \mathbf{Q}_{i} \cdot \phi_{i j} \cdot \mathbf{Q}_{j}, \\
\phi_{i j}=\phi_{\mu \nu}\left(R_{i j}\right)=\frac{\delta_{\mu \nu}}{R_{i j}^{3}}-\frac{3 R_{i j, \mu} R_{i j, \nu}}{R_{i j}^{5}} .
\end{gathered}
$$

The lattice transform of this interaction is ${ }^{15}$

$$
\begin{gathered}
T_{\mu \nu}(\mathbf{k})=\sum_{j \neq 0} e^{i \mathbf{k} \cdot \mathbf{R}_{j}} \phi_{\mu \nu}\left(\mathbf{R}_{j}\right), \\
\lim _{k a \ll 1} T_{\mu \nu}(\mathbf{k})=-\frac{4 \pi}{\Omega_{0}}\left[\frac{k_{\mu} k_{\nu}}{k^{2}}-\frac{\delta_{\mu \nu}}{3}\right],
\end{gathered}
$$

where $\Omega_{0}$ is the volume of the unit cell. We will usually take the limit of the small wave vector in these expressions.

\section{CHARGED DONOR}

First, we evaluate the energy of an isolated donor without an electron nearby. We transform the vibrations to collective coordinates,

$$
\mathbf{Q}_{j}=\frac{1}{\sqrt{N}} \sum_{\mathbf{k}} e^{i \mathbf{R}_{j} \cdot \mathbf{k}} \mathbf{Q}_{\mathbf{k}} .
$$

The potential-energy terms for longitudinal-optical (LO) phonons are

$$
\begin{gathered}
V=\sum_{\mathbf{k}}\left[\frac{K_{L}}{2}\left(\hat{k} \cdot \mathbf{Q}_{k}\right)^{2}+\beta_{k} \hat{k} \cdot \mathbf{Q}_{k}\right], \\
K_{L}=K+\frac{2}{3} \frac{\left(e^{*}\right)^{2}}{\Omega_{0} \varepsilon(\infty)} .
\end{gathered}
$$

The second term in $K_{L}$ comes from the dipole-dipole interaction. The lattice transform of $V_{p d}$ is

$$
\begin{gathered}
V_{p d}=-\frac{q e^{*}}{4 \pi \varepsilon(\infty) \sqrt{N}} \sum_{\mathbf{k}} \mathbf{Q}_{k} \cdot \mathbf{W}_{\mathbf{k}} \sum_{l} e^{i \mathbf{k} \cdot \mathbf{R}_{l}}, \\
\mathbf{W}_{\mathbf{k}}=\sum_{j \neq 0} e^{i \mathbf{k} \cdot \mathbf{R}_{j}} \frac{\mathbf{R}_{j}}{R_{j}^{3}} \approx \frac{4 \pi i}{\Omega_{o}} \frac{\mathbf{k}}{k^{2}},
\end{gathered}
$$

where the latter limit is for long wavelength. In this case, the expression for $\beta_{\mathbf{k}}$ is

$$
\beta_{k}=-\frac{q e^{*} i}{\varepsilon(\infty) k \Omega_{0} \sqrt{N}} \sum_{l} e^{i \mathbf{k} \cdot \mathbf{R}_{l}} .
$$

The phonon displacements enter as $\left(\hat{k} \cdot \mathbf{Q}_{k}\right)$, where the notation emphasizes that only longitudinal-optical phonons are involved. The transverse- $\left(\omega_{T O}\right)$ and longitudinal- $\left(\omega_{L O}\right)$ optical-phonon frequencies are defined as

$$
\begin{gathered}
\omega_{T O}^{2} \equiv \frac{K_{T}}{M}=\frac{K}{M}-\frac{1}{3} \omega_{i}^{2}, \\
\omega_{L O}^{2}=\frac{K_{L}}{M}=\frac{K}{M}+\frac{2}{3} \omega_{i}^{2}, \quad \omega_{i}^{2}=\frac{\left(e^{*}\right)^{2}}{M \Omega_{0} \varepsilon(\infty)} .
\end{gathered}
$$

The static dielectric function is

$$
\varepsilon(0)=\varepsilon(\infty)\left[1+\frac{\omega_{i}^{2}}{\omega_{T O}^{2}}\right] .
$$

We complete the square on the interaction in Eq. (11),

$$
V=\frac{K_{L}}{2} \sum_{\mathbf{k}}\left[\hat{k} \cdot \mathbf{Q}_{k}+\frac{\beta_{k}}{K_{L}}\right]^{2}-\frac{1}{2 K_{L}} \sum_{\mathbf{k}}\left|\beta_{k}\right|^{2} .
$$

The last term is the effective interaction between the optical phonons and the donor due to the phonon polarization,

$$
\begin{aligned}
V^{\prime} & =-\frac{1}{2 K_{L}} \sum_{\mathbf{k}}\left|\beta_{k}\right|^{2} \\
& =-\frac{q^{2}\left(e^{*}\right)^{2}}{2 K_{L} \varepsilon(\infty)^{2} \Omega_{0}} \int \frac{d^{3} k}{(2 \pi)^{3}} \frac{1}{k^{2}} \sum_{l l^{\prime}} e^{i \mathbf{k} \cdot\left(\mathbf{R}_{l}-\mathbf{R}_{l^{\prime}}\right)} .
\end{aligned}
$$

For $\mathbf{R}_{l} \neq \mathbf{R}_{l^{\prime}}$, the wave-vector integral gives the effective interaction between the two donors,

$$
V_{l l^{\prime}}^{\prime}=-\frac{q^{2}}{4 \pi \varepsilon(\infty)} \frac{\omega_{i}^{2}}{\omega_{L O}^{2}} \frac{1}{\left|\mathbf{R}_{l}-\mathbf{R}_{l^{\prime}}\right|} .
$$

We combine this term with the direct interaction in Eq. (3) to find

$$
\begin{gathered}
V_{l l^{\prime}}=\frac{q^{2}}{4 \pi \varepsilon(\infty)}\left[1-\frac{\omega_{i}^{2}}{\omega_{L O}^{2}}\right] \frac{1}{\left|\mathbf{R}_{l}-\mathbf{R}_{l^{\prime}}\right|}, \\
V_{l l^{\prime}}=\frac{q^{2}}{4 \pi \varepsilon(0)} \frac{1}{\left|\mathbf{R}_{l}-\mathbf{R}_{l^{\prime}}\right|}, \\
\frac{1}{\varepsilon(0)}=\frac{1}{\varepsilon(\infty)}\left[1-\frac{\omega_{i}^{2}}{\omega_{L O}^{2}}\right] .
\end{gathered}
$$

The latter identity follows directly from Eq. (18). The effective Coulomb interaction between ionized donors is screened by the static dielectric function.

The case of $l=l^{\prime}$ gives the energy of a single donor from the phonon polarization. It has the approximate value of

$$
\begin{gathered}
\int \frac{d^{3} k}{(2 \pi)^{3}} \frac{1}{k^{2}}=\frac{4 \pi}{(2 \pi)^{3}} \frac{\pi}{\tilde{a}}=\frac{1}{2 \pi \tilde{a}}, \\
V^{\prime}=-\frac{q^{2}\left(e^{*}\right)^{2}}{4 \pi \tilde{a} K_{L} \varepsilon(\infty)^{2} \Omega_{0}},
\end{gathered}
$$

where $\tilde{a}$ is approximately a lattice constant. An exact expression for $\tilde{a}$ is given below. This energy term comes from the polarization induced by a single charged donor, which reduces its energy. 


\section{ELECTRON BOUND TO DONOR}

We repeat the above calculation but include an electron bound to a donor at $\mathbf{R}_{l}$. We do this calculation of the donor binding energy using a variational parameter $\alpha$ defined as

$$
\begin{gathered}
\psi(r)=\sqrt{\frac{\alpha^{3}}{\pi a_{0}^{3}}} \exp \left[-\alpha r / a_{0}\right], \\
n(r)=|\psi(r)|^{2},
\end{gathered}
$$

where the Bohr radius is given by

$$
a_{0}=4 \pi \varepsilon(\infty) \frac{\hbar^{2}}{e^{2} m^{*}},
$$

and $m^{*}$ is the band effective mass of the conduction electron. Larsen $^{1}$ did a similar calculation but omitted the interaction $V_{p d}$, which is the term that polarizes the lattice in the absence of an electron. The inclusion of this important term is crucial for including all essential interactions. Again, we transform the vibrations to collective coordinates,

$$
\mathbf{Q}_{j}=\frac{1}{\sqrt{N}} \sum_{\mathbf{k}} e^{i \mathbf{R}_{j} \cdot \mathbf{k}} \mathbf{Q}_{\mathbf{k}} .
$$

The potential-energy terms in the limit of long wavelength are

$$
\begin{gathered}
V=-\sum_{\mathbf{k}}\left[\frac{K_{L}}{2}\left(\hat{k} \cdot Q_{k}\right)^{2}+\gamma_{k} \hat{k} \cdot \mathbf{Q}_{k}\right], \\
\gamma_{k}=\frac{i e^{*}}{\varepsilon(\infty) k \Omega_{0} \sqrt{N}}\left[q+e \Lambda\left(k a_{0} / 2 \alpha\right)\right] \sum_{l} e^{i \mathbf{k} \cdot \mathbf{R}_{l},} \\
\Lambda(x)=\frac{1}{\left(1+x^{2}\right)^{2}} .
\end{gathered}
$$

The first term in $\gamma_{k}$ comes from the donor-phonon interaction, and the second term comes from the electron-phonon interaction. The donor-phonon interaction is evaluated in the long-wavelength limit. We complete the square on the interaction, and also set $q=-e$,

$$
V=\frac{K_{L}}{2} \sum_{\mathbf{k}}\left[\hat{k} \cdot \mathbf{Q}_{k}+\frac{\gamma_{k}}{K_{L}}\right]^{2}-\frac{1}{2 K_{L}} \sum_{\mathbf{k}}\left|\gamma_{k}\right|^{2} .
$$

The last term is part of the effective interaction between the electron and the donor due to the phonon polarization,

$$
\begin{gathered}
V^{\prime \prime}=-\frac{1}{2 K_{L}} \sum_{\mathbf{k}}\left|\gamma_{k}\right|^{2}, \\
V^{\prime \prime}=-\frac{e^{2}\left(e^{*}\right)^{2}}{2 K_{L} \varepsilon(\infty)^{2} \Omega_{0}} \int \frac{d^{3} k}{(2 \pi)^{3}} \frac{1}{k^{2}} \\
\times\left[1-\Lambda\left(k a_{0} / 2 \alpha\right)\right]^{2} \sum_{l l^{\prime}} e^{i \mathbf{k} \cdot\left(\mathbf{R}_{l}-\mathbf{R}_{l^{\prime}}\right)} .
\end{gathered}
$$

We evaluate this expression below in several limits. There are two types of terms. If $l \neq l^{\prime}$, then it is the phonon-induced interaction between two neutral donors. If $l=l^{\prime}$, then it is the polaron correction to the binding energy of a single donor. Note that $\Lambda(x=0)=1$ so the integrand vanishes at $k=0$. There is no long-range Coulomb interaction between neutral donors.

\section{A. Interactions between donors}

First, consider the case of $l \neq l^{\prime}$ so the expression gives the phonon-induced interaction energy between two neutral donors. For the effective mass limit, where the orbit radius $a_{0}$ covers many lattice constants, we can extend the limit of integration to infinity. We change to dimensionless variables $x=k a_{0} /(2 \alpha), y=2 \alpha R_{l l^{\prime}} / a_{0}$, and we get

$$
\begin{gathered}
V_{p p^{\prime}}^{\prime \prime}=-\frac{e^{2}}{4 \pi \varepsilon(\infty) a_{0}} \frac{\omega_{i}^{2}}{\Omega_{L O}^{2}} I(y), \\
I(y)=\frac{2}{\pi y} \int_{0}^{\infty} d x \sin (x y) \frac{x^{3}\left(2+x^{2}\right)^{2}}{\left(1+x^{2}\right)^{4}} .
\end{gathered}
$$

The above integral can be evaluated by contour integration. It gives the term

$$
I(y)=\frac{e^{-y}}{y} \sum_{m=0}^{3} a_{m} y^{m} .
$$

The interaction decays exponentially, which is typical of the interaction between neutral-charge distributions when neglecting correlations.

\section{B. Neutral donor number 1}

We evaluate Eq. (37) for the case of $l=l^{\prime}$. We expand the factor as

$$
(1-\Lambda)^{2}=1-2 \Lambda+\Lambda^{2}
$$

We evaluate the first term ("1") as Eq. (27), so the polarization energy of the donor is unchanged. The other two terms contribute to the donor binding energy. In the effective mass limit, the last two terms are evaluated assuming that the $k$ integral goes to infinity,

$$
\frac{4 \pi}{(2 \pi)^{3}} \int_{0}^{\infty} d k\left[-2 \Lambda+\Lambda^{2}\right]=-\frac{\alpha}{2 \pi a_{0}}\left[1-\frac{5}{16}\right]=-\frac{11 \alpha}{32 \pi a_{0}} .
$$

The variational energy for the donor binding is

$$
\mathcal{E}(\alpha)=E_{R y}\left[\alpha^{2}-2 \alpha(1-\lambda)\right], \quad \lambda=\frac{11}{16} \frac{\omega_{i}^{2}}{\omega_{L O}^{2}} .
$$

Minimizing with respect to $\alpha$ gives

$$
\begin{gathered}
\alpha_{0}=1-\lambda=\frac{5}{16}+\frac{11}{16} \frac{\varepsilon(\infty)}{\varepsilon(0)}, \\
\mathcal{E}\left(\alpha_{0}\right)=-E_{R y}(1-\lambda)^{2} .
\end{gathered}
$$

For weakly bound donors, the dielectric screening is not given by either $\varepsilon_{\infty}$ or $\varepsilon(0)$. Instead, it is $5 / 16$ of $\varepsilon_{\infty}$ and $11 / 16$ of $\varepsilon(0)$.

An interesting result is obtained if we neglect $\Lambda^{2}$. In that case, the total variational energy for the electron bound to the donor is

$$
\begin{gathered}
\mathcal{E}(\alpha)=\frac{\hbar^{2} \alpha^{2}}{2 m^{*} a_{0}^{2}}-\frac{e^{2} \alpha}{4 \pi \varepsilon(\infty) a_{0}}\left(1-\frac{\omega_{i}^{2}}{\omega_{L O}^{2}}\right), \\
\mathcal{E}(\alpha)=\frac{\hbar^{2} \alpha^{2}}{2 m^{*} a_{0}^{2}}-\frac{e^{2} \alpha}{4 \pi \varepsilon(0) a_{0}} .
\end{gathered}
$$


Varying $\alpha$ gives the minimum,

$$
\begin{gathered}
\alpha_{0}=\left[\frac{\varepsilon(\infty)}{\varepsilon(0)}\right], \\
\mathcal{E}\left(\alpha_{0}\right)=-E_{R y}\left[\frac{\varepsilon(\infty)}{\varepsilon(0)}\right]^{2} .
\end{gathered}
$$

The donor binding energy is screened by the zero-frequency dielectric function, which includes the contribution from the optical phonons. This is the usual form of the SHM, which is obtained only by neglecting $\Lambda^{2}$. Since neglecting $\Lambda^{2}$ is a poor approximation, the SHM is not accurate.

\section{Neutral donor number 2}

Here we evaluate the screening corrections to the neutral donor by a different method, which calculates all energy terms in real space. This has the advantage of not having to make a long-wavelength approximation. The final expression should be more accurate.

The interaction $V_{e p}$ is rewritten as

$$
\begin{aligned}
& V_{e p}=\frac{e e^{*}}{4 \pi \varepsilon(\infty) \sqrt{N}} \sum_{\mathbf{k}}\left(\sum_{l} e^{i \mathbf{k} \cdot \mathbf{R}_{l}}\right) \\
& \times \sum_{j \neq 0} e^{i \mathbf{k} \cdot \mathbf{R}_{j}} \mathbf{Q}_{\mathbf{k}} \cdot \vec{\nabla}_{R_{j}} f\left(R_{j}\right), \\
& f(R)=\int d^{3} r \frac{n(r)}{|\mathbf{R}-\mathbf{r}|}=\frac{1}{R}-\frac{1}{R} e^{-2 \alpha R / a_{0}}\left(1+\frac{\alpha R}{a_{0}}\right) .
\end{aligned}
$$

When $q=-e$, the $1 / R$ term cancels $V_{p d}$. The combined terms give, for $\gamma_{\mathbf{k}}$,

$$
\begin{aligned}
\vec{\gamma}_{\mathbf{k}}= & -\frac{e e^{*}}{4 \pi \varepsilon(\infty) \sqrt{N}}\left(\sum_{l} e^{i \mathbf{k} \cdot \mathbf{R}_{l}}\right) \sum_{j \neq 0} e^{i \mathbf{k} \cdot \mathbf{R}_{j}} \vec{\nabla}_{R} \\
& \times\left\{\frac{e^{-2 \alpha R / a_{0}}}{R}\left(1+\alpha R / a_{0}\right)\right\}_{R=R_{j}} \\
= & \frac{e e^{*}}{4 \pi \varepsilon(\infty) \sqrt{N}}\left(\sum_{l} e^{i \mathbf{k} \cdot \mathbf{R}_{l}}\right) \sum_{j \neq 0} e^{i \mathbf{k} \cdot \mathbf{R}_{j}} \hat{R}_{j} \frac{e^{-2 \alpha R_{j} / a_{0}}}{R_{j}^{2}} \\
& \times\left(1+2 \alpha R_{j} / a_{0}+2 \alpha^{2} R_{j}^{2} / a_{0}^{2}\right) .
\end{aligned}
$$

The next step is to take $\sum_{\mathbf{k}}\left|\gamma_{\mathbf{k}}\right|^{2}$. There is a double sum over $\left(j, j^{\prime}\right)$. When $l=l^{\prime}$, the summation over $\mathbf{k}$ forces $\mathbf{R}_{j}=\mathbf{R}_{j^{\prime}}$. The final answer for $l=l^{\prime}$ is

$$
\begin{aligned}
V_{p p}^{\prime \prime}= & -\frac{e^{2}\left(e^{*}\right)^{2}}{2 K_{L}[4 \pi \varepsilon(\infty)]^{2}} \sum_{j \neq 0} \frac{e^{-4 \alpha R_{j} / a_{0}}}{R_{j}^{4}} \\
& \times\left(1+2 \alpha R_{j} / a_{0}+2 \alpha^{2} R_{j}^{2} / a_{0}^{2}\right)^{2} .
\end{aligned}
$$

This answer is only slightly different than case number 1 . The summation over $\mathbf{R}_{j}$ converges to a finite value. There is no ionized donor energy as in Eq. (27). That is sensible, since the neutral donor should not attract long-range polarization.
We rewrite the above expression using the nearest-neighbor distance $d$ as a unit of length,

$$
\begin{aligned}
V_{p p}^{\prime \prime}= & -E_{R y} \frac{\omega_{i}^{2}}{\omega_{L O}^{2}} \frac{a_{0}}{d} F\left(\alpha d / a_{0}\right), \\
F\left(\alpha d / a_{0}\right)= & \frac{\Omega_{0} d}{4 \pi} \sum_{j \neq 0} \frac{e^{-4 \alpha R_{j} / a_{0}}}{R_{j}^{4}} \\
& \times\left(1+2 \alpha R_{j} / a_{0}+2 \alpha^{2} R_{j}^{2} / a_{0}^{2}\right)^{2} .
\end{aligned}
$$

An important result is the screening energy of the ionized donor, which is found by setting $a_{0}=\infty$,

$$
V^{\prime}=-\frac{e^{2}}{8 \pi \varepsilon(\infty) d}\left[\frac{\left(e^{*}\right)^{2}}{\varepsilon(\infty) K_{L} \Omega_{0}}\right] F(0) .
$$

This formula is exact and is an improvement to Eq. (27). For the fcc lattice, $F(0)=1.425780$ using Ewald methods, which are described in the Appendix. By comparing the two expressions, we determine that

$$
\tilde{a}=a \frac{\sqrt{2}}{F(0)}=0.992 a .
$$

The energy of the ionized donor must be subtracted from the result of Eq. (53), which gives the final donor binding energy,

$$
\begin{gathered}
\mathcal{E}(\alpha)=E_{R y}\left\{\alpha^{2}-2 \alpha-\eta\left[F\left(\alpha d / a_{0}\right)-F(0)\right]\right\}, \\
\eta=\frac{\left(e^{*}\right)^{2} a_{0}}{\varepsilon(\infty) K_{L} d \Omega_{0}}=\frac{a_{0}}{d} \frac{\omega_{i}^{2}}{\omega_{L O}^{2}} .
\end{gathered}
$$

For a given crystal structure, $F(x)$ is a function of $x=\alpha d / a_{0}$. The self-consistent variational equation for the coupling constant $\alpha_{0}$ at minimum energy is

$$
\alpha_{0}=1+\frac{1}{2} \frac{\omega_{i}^{2}}{\omega_{L O}^{2}} F^{\prime}(x)_{x=\alpha_{0} d / a_{0}},
$$

where $F^{\prime}(x)=d F / d x<0$.

For many semiconductors, the value of $x$ is small, and it is adequate to take the limit of small $x$,

$$
F(x)-F(0)=x F^{\prime}(0)+O\left(x^{3}\right) .
$$

Using Ewald methods, we show for the fcc lattice that $F^{\prime}(0)=-11 / 8$, which makes Eq. (57) identical to Eq. (43). The derivation in the Appendix suggests that this result $\left[F^{\prime}(0)=-11 / 8\right]$ is valid for all lattices. For weakly bound donors, our two derivations give the same result. Equations (57) and (59) are useful for materials with larger values of $x$.

\section{Atomic limit for donor}

One case is when the donor is tightly bound, so that $d / a_{0}>1$. Even then, the term $F(x)$ is not small and cannot be neglected. For example, $F(1)=0.35$.

\section{E. Ferroelectrics}

Many ferroelectrics have the feature in which as one nears the transition temperature $T_{c}, \varepsilon(0)$ diverges, while $\varepsilon(\infty)$ remains constant. This is the result of $\omega_{T O}$ going to zero, while $\omega_{L O}$ remains constant. In our first model, as $\omega_{T O} \rightarrow 0$, then $\omega_{L O} \rightarrow \omega_{i}$. The donor binding energy does not vanish, but is given by $E_{R y}(5 / 16)^{2} \approx 0.1 E_{R y}$. 


\section{FCC LATTICE}

We present some calculations for the fcc lattice, where $d=$ $a / \sqrt{2}$ and $\Omega_{0}=a^{3} / 4=d^{3} / \sqrt{2}$. We discuss the evaluation of the function $F(x)$, in Eq. (54), and its derivative $F^{\prime}(x)$,

$$
F(x)=\frac{\Omega_{0} d}{4 \pi} \sum_{j \neq 0} \frac{e^{-4 x R_{j} / d}}{R_{j}^{4}}\left[1+2 x R_{j} / d+2 x^{2}\left(R_{j} / d\right)^{2}\right]^{2} .
$$

We define the summation variable as $y_{j}=R_{j} / d$, which starts out as one for the 12 nearest neighbors,

$$
F(x)=\frac{1}{4 \pi \sqrt{2}} \sum_{j \neq 0} \frac{e^{-4 x y_{j}}}{y_{j}^{4}}\left(1+2 x y_{j}+2 x^{2} y_{j}^{2}\right)^{2} .
$$

One way to evaluate Eq. (62) is to sum all neighbors out to a distance $y_{x}$. However, the expression converges very slowly for small values of $x$. We performed the computer summations out to a distance $y_{x}$ and noticed that they seem to scale as $-1 / y_{x}$. We did a least-squares fit to the expression $F(0)=C-B / y_{x}$, which gave $C=1.425781$, which is very close to the Ewald result. Using Ewald methods in the Appendix, we obtain the same value.

A similar process determines $F^{\prime}(x)$ :

$$
F^{\prime}(x)=-\frac{8 x^{2}}{4 \pi \sqrt{2}} \sum_{j \neq 0} \frac{e^{-4 x y_{j}}}{y_{j}}\left(1+2 x y_{j}+2 x^{2} y_{j}^{2}\right) .
$$

Using Ewald methods, we found the exact expression $F^{\prime}(0)=$ $-11 / 8$. In the Appendix, we derive the next terms in the power series in $x$,

$$
F(x)=F(0)-\frac{11}{8} x+\frac{B}{3} x^{3}+O\left(x^{4}\right)
$$

where $B=1.4595$. With these values, we constructed the graph of $F(x)$ shown in Fig. 1.

The eigenvalue equation (59) involves the function $F^{\prime}(x)$, which we have evaluated from Eq. (63). For fitting purposes, it is useful to have an analytic function that approximates

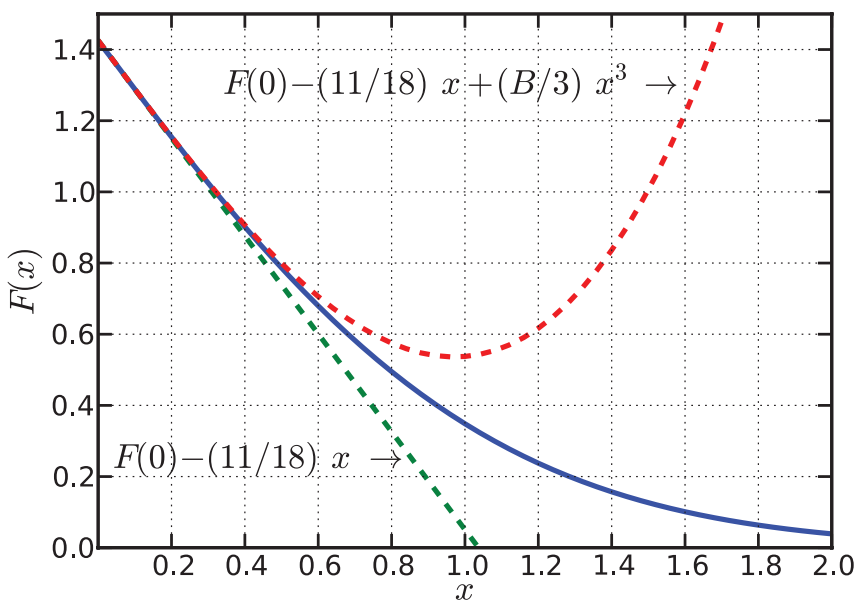

FIG. 1. (Color online) $F(x)$ for the fcc lattice. The lower dashed curve shows the two first terms of the expansion in $x$, while the upper curve shows the results of the first four terms in $x$.
TABLE I. Donor binding energies in meV. Only cubic crystals are included. Data from Ref. 18.

\begin{tabular}{llcccccc}
\hline \hline & & & & & $E_{D}$ & $E_{D}$ & $E_{D}$ \\
Crystal & $m^{*}$ & $\varepsilon(\infty)$ & $\varepsilon(0)$ & $E_{R y}$ & $(\mathrm{SHM})$ & $($ Theory) & (Expt.) \\
\hline $\mathrm{GaAs}$ & 0.0665 & 10.9 & 12.5 & 7.6 & 5.8 & 6.3 & $5.8-5.9$ \\
$\mathrm{GaSb}$ & 0.0396 & 14.4 & 15.7 & 2.6 & 2.3 & 2.4 & \\
$\mathrm{InP}$ & 0.079 & 9.6 & 12.6 & 11.7 & 6.8 & 8.2 & 7.1 \\
$\mathrm{InAs}$ & 0.023 & 12.25 & 15.15 & 2.1 & 1.4 & 1.6 & \\
$\mathrm{InSb}$ & 0.0145 & 15.68 & 17.5 & 0.80 & 0.60 & 0.69 & 0.7 \\
$\mathrm{ZnS}$ & 0.27 & 5.2 & 8.9 & 136 & 46 & 69 & 30 \\
$\mathrm{ZnSe}$ & 0.16 & 6.0 & 8.8 & 60.4 & 28.1 & 36.9 & $25-29$ \\
$\mathrm{ZnTe}$ & 0.12 & 7.3 & 10.0 & 30.6 & 16.3 & 20.3 & 18.3 \\
$\mathrm{CdTe}$ & 0.09 & 7.4 & 10.6 & 22.3 & 10.9 & 14.0 & 22.0 \\
$\mathrm{MgO}$ & 0.35 & 3.0 & 9.9 & 313 & 48.6 & 113 & \\
$\mathrm{CaO}$ & 0.50 & 3.1 & 12.1 & 708 & 69 & 248 & 3100 \\
$\mathrm{SrO}$ & 0.54 & 3.5 & 16.2 & 600 & 191 & 401 & 2600 \\
$\mathrm{BaO}$ & 0.59 & 3.56 & 37.4 & 633 & 147 & 394 & 2000 \\
\hline
\end{tabular}

these numerical values. The following expression is accurate to $O(0.3 \%)$ :

$$
\begin{aligned}
F^{\prime}(x) \approx & -\frac{24 x^{2}}{\pi \sqrt{2}}\left[e^{-4 x}\left(1+2 x+2 x^{2}\right)-e^{-1.3 \xi x}\right]+B x^{2} e^{-\xi x} \\
& -\frac{11}{8} e^{-\xi x}\left[1+\xi x+\frac{1}{2}(\xi x)^{2}\right],
\end{aligned}
$$

where $B$ is defined above, and the fitting parameter is $\xi=$ 5.09490. The first term on the right is the asymptotic limit from the nearest-neighbor contribution in Eq. (63).

We have used our theory to calculate the donor binding energies in several crystals, as shown in Table I. The data are from Landolt-Börnstein. ${ }^{18}$ Only cubic crystals are included, and only those whose conduction-band minimum is at the center of the Brillouin zone, so the effective mass is isotropic. For the oxides, the binding is sufficiently large that we solved Eq. (59) self-consistently. This gives a much larger binding energy compared to the SHM. Both our theory and the SHM are poor for the oxides, since electrons are too tightly bound for effective mass theory to be valid. We show these results only to demonstrate that for large values of $x$, our theory is different than the SHM.

As stated in Sec. I, we regard our theory as a strong-coupling result, which is valid when the two dielectric functions $[\varepsilon(\infty)$ and $\varepsilon(0)]$ are very different. This is not the case for most III-V semiconductors, and our theory does not do well for these materials. However, we expect our theory to apply quite well to the II-VI semiconductors, which are generally more polar than the III-V semiconductors. Table I shows our theory does relatively well for these materials.

\section{DISCUSSION}

We have evaluated the polaron corrections to the binding energy of electrons to donors. We include the following interactions: electron-phonon, donor-phonon, and phononphonon. Two variational calculations gave identical results in the limit of a small binding energy. For the case of a large binding energy, one method is exact but gives an equation for the variational parameter that must be solved self-consistently. 
Most textbooks give the donor binding energy as

$$
E_{D}=-\frac{e^{4} m}{2[4 \pi \varepsilon(0)]^{2} \hbar^{2}},
$$

where $m$ is the band effective mass of the electron and $\varepsilon(0)$ is the static dielectric function. We get a different expression,

$$
\begin{gathered}
E_{D}=-\frac{e^{4} m}{2[4 \pi \tilde{\varepsilon}]^{2} \hbar^{2}}, \\
\frac{1}{\tilde{\varepsilon}}=\frac{5}{16 \varepsilon(\infty)}+\frac{11}{16 \varepsilon(0)},
\end{gathered}
$$

which includes both dielectric constants $[\varepsilon(\infty), \varepsilon(0)]$. The effective Bohr radius is $\tilde{a}_{0}=4 \pi \tilde{\varepsilon} \hbar^{2} / m e^{2}$. We expect our theory applies in the strong-coupling limit, when $\varepsilon(\infty)$ and $\varepsilon(0)$ are very different.

There is also the question of whether the effective mass $m^{*}$ includes polaron corrections or is just the bare band mass. In our theory, it is the bare band mass. The above equation applies only to the case of $E_{D}<\hbar \omega_{L O}$. For materials with larger binding energies, one must solve a nonlinear equation to determine the parameters of the binding energy. We used our theory to calculate the donor binding energy of several materials with the fcc crystal structure.

One might consider a similar calculation for the electronic polaron effects: the terms that make $\varepsilon(\infty)$ differ from $\varepsilon_{0}$. Here one would start with a bare interaction, screened by the vacuum dielectric constant $\varepsilon_{0}$, and consider how the electronic screening changes the donor binding energy. That is a different calculation than we have done here, since the electronic screening is in different places in the crystal. For the oxides, and other ionic crystals, the electronic polarization resides mostly with the anions. ${ }^{19}$ However, for covalent materials, it resides in the bonds between ions.

\section{ACKNOWLEDGMENTS}

One of us (G.D.M.) thanks the Erasmus Mundus program of the European Union for financial support. He also thanks Professors Per Hyldgaard and Elsebeth Schröder for support while at Chalmers Technical University in Göteborg.

\section{APPENDIX: EWALD SUMMATION}

The functions $F(x), F^{\prime}(x)$ are evaluated using an Ewald method. ${ }^{20}$ We start with an evaluation of $F(0)$ since it is easy:

$$
\begin{gathered}
F(0)=\frac{\Omega_{0} d}{4 \pi} \sum_{j \neq 0} \frac{1}{R_{j}^{4}}=2 \frac{\Omega_{0} d}{4 \pi} \sum_{j \neq 0} \int_{0}^{\infty} d t t^{3} e^{-t^{2} R_{j}^{2}} \\
=F_{L}+F_{R}, \\
F_{L}=2 \frac{\Omega_{0} d}{4 \pi} \sum_{j \neq 0} \int_{\eta}^{\infty} d t t^{3} e^{-t^{2} R_{j}^{2}} \\
=\frac{\Omega_{0} d}{4 \pi} \sum_{j \neq 0} \frac{1}{R_{j}^{4}} e^{-\left(\eta R_{j}\right)^{2}}\left[1+\left(\eta R_{j}\right)^{2}\right], \\
F_{R}=2 \frac{\Omega_{0} d}{4 \pi} \sum_{j \neq 0} \int_{0}^{\eta} d t t^{3} e^{-t^{2} R_{j}^{2}},
\end{gathered}
$$

where $\eta=C / d$. The term $F_{L}$ we leave as is, since it converges rapidly in real space. The term $F_{R}$ is changed to a summation over reciprocal lattice vectors by creating a periodic function
TABLE II. Ewald summation for $F(0)$.

\begin{tabular}{lccc}
\hline \hline $\mathrm{C}$ & $F_{L}$ & $F_{R}$ & $F$ \\
\hline 0.7 & 0.812176 & 0.613604 & 1.425780 \\
0.9 & 0.646635 & 0.779145 & 1.425780 \\
1.1 & 0.492121 & 0.933659 & 1.425780 \\
1.3 & 0.353968 & 1.071812 & 1.425780 \\
\hline \hline
\end{tabular}

of position $\mathbf{r}$,

$$
\begin{gathered}
V_{R}(\mathbf{r})=2 \sum_{j} \int_{0}^{\eta} d t t^{3} e^{-t^{2}\left(\mathbf{R}_{j}-\mathbf{r}\right)^{2}}=\sum_{\mathbf{G}} v(\mathbf{G}) e^{i \mathbf{G} \cdot \mathbf{r}} \\
v(\mathbf{G})=\frac{2}{\Omega_{0}} \int_{0}^{\eta} t^{3} d t \int d^{3} r e^{-i \mathbf{G} \cdot \mathbf{r}} e^{-t^{2} r^{2}} \\
v(\mathbf{G})=\frac{2 \pi^{3 / 2}}{\Omega_{0}} \int_{0}^{\eta} d t e^{-G^{2} / 4 t^{2}}=\frac{G \pi^{3 / 2}}{\Omega_{0}} \int_{0}^{2 \eta / G} d s e^{-1 / s^{2}} \\
F_{R}=\frac{\Omega_{0} d}{4 \pi}\left[\frac{2 \pi^{3 / 2} \eta}{\Omega_{0}}+\sum_{\mathbf{G} \neq 0} v(\mathbf{G})-\frac{\eta^{4}}{2}\right]
\end{gathered}
$$

The first term in $F_{R}$ is the $v(0)$, and the last term is from the subtraction of the $\mathbf{R}_{j}=0$ term from $V_{R}(0)$. Table II shows the separate contributions as a function of $C$. The final result is independent of $C$, which is a good way to check the computer code.

Next, we determine $F^{\prime}(0)$ starting from Eq. (63). Since the prefactor is proportional to $x^{2}$, we must take the limit of $x \rightarrow 0$ with some care. We use Ewald's method with an exponential,

$$
\begin{gathered}
\frac{1}{y_{j}}=\int_{0}^{\infty} d t e^{-t y_{j}}=\int_{0}^{\eta} d t e^{-t y_{j}}+\int_{\eta}^{\infty} d t e^{-t y_{j}}, \text { (A9) } \\
F_{L}^{\prime}(x)=-\frac{8 x^{2}}{4 \pi \sqrt{2}} \sum_{j \neq 0} \frac{e^{-y_{j}(4 x+\eta)}}{y_{j}}\left(1+2 x y_{j}+2 x^{2} y_{j}^{2}\right), \\
F_{R}^{\prime}(x)=-\frac{8 x^{2}}{4 \pi \sqrt{2}} \int_{0}^{\eta} d t \sum_{j \neq 0} e^{-y_{j}(4 x+t)}\left(1+2 x y_{j}+2 x^{2} y_{j}^{2}\right) .
\end{gathered}
$$

The lattice sum vanishes at $x=0: F_{L}^{\prime}(0)=0$. We evaluate $F_{R}^{\prime}(x)$ by constructing a periodic function of $\mathbf{r}$ and then determining its Fourier coefficient,

$$
\begin{gathered}
V_{R}(\mathbf{r})=\sum_{j} e^{-\left|\mathbf{y}_{j}-\mathbf{r}\right|(4 x+t)}\left[1+2 x\left|\mathbf{y}_{j}-\mathbf{r}\right|+2 x^{2}\left(\mathbf{y}_{j}-\mathbf{r}\right)^{2}\right] \\
=\sum_{\mathbf{G}} e^{i \mathbf{G} \cdot \mathbf{r}} v(G), \\
\begin{aligned}
& v(G)= \frac{1}{\Omega_{y}} \int d^{3} r \exp [-i \mathbf{G} \cdot \mathbf{r}-r(4 x+t)] \\
& \times\left[1+2 x r+2 x^{2} r^{2}\right], \\
& v(G)= 8 \pi \sqrt{2}\left[\frac{\tau}{\left(\tau^{2}+G^{2}\right)^{2}}+2 x \frac{3 \tau^{2}-G^{2}}{\left(\tau^{2}+G^{2}\right)^{3}}\right. \\
&\left.+24 x^{2} \tau \frac{\tau^{2}-G^{2}}{\left(\tau^{2}+G^{2}\right)^{4}}\right], \\
& \tau=4 x+t .
\end{aligned}
\end{gathered}
$$


The result for $F^{\prime}(0)$ comes from the term with $\mathbf{G}=0$. This integral is easy to evaluate:

$$
\begin{aligned}
v(0) & =8 \pi \sqrt{2}\left[\frac{1}{\tau^{3}}+\frac{6 x}{\tau^{4}}+\frac{24 x^{2}}{\tau^{5}}\right], \\
\int_{0}^{\eta} d t v(0) & =8 \pi \sqrt{2}\left[\frac{1}{2}\left(\frac{1}{16 x^{2}}-\frac{1}{(4 x+\eta)^{2}}\right)+2 x\left(\frac{1}{(4 x)^{3}}-\frac{1}{(4 x+\eta)^{3}}\right)+6 x^{2}\left(\frac{1}{(4 x)^{4}}-\frac{1}{(4 x+\eta)^{4}}\right)\right] .
\end{aligned}
$$

The terms in the above equation, which are proportional to $O\left(x^{-2}\right)$, give the limit as $x \rightarrow 0$,

$$
F^{\prime}(0)=-\frac{8}{16}\left(1+1+\frac{3}{4}\right)=-\frac{11}{8} .
$$

One can also use the Ewald sum to find the first nonzero term in $x$, which is

$$
\begin{aligned}
F^{\prime}(x) & =-\frac{11}{8}+B x^{2}+O\left(x^{3}\right), \\
B & =-\frac{\sqrt{2}}{\pi} \sum_{j \neq 0} \frac{e^{-y_{j} \eta}}{y_{j}}+\frac{8}{\eta^{2}}+\frac{8 \eta}{4 \pi \sqrt{2}}-8 \sum_{\mathbf{G} \neq 0} \frac{\eta^{2}}{G^{2}\left(G^{2}+\eta^{2}\right)},
\end{aligned}
$$

which gives $B=1.4595 \pm 0.0001$.

${ }^{1}$ D. M. Larsen, Phys. Rev. 187, 1147 (1969).

${ }^{2}$ A. M. Stoneham, J. Phys. C 3, L131 (1970).

${ }^{3}$ D. M. Larsen, J. Phys. C 3, L185 (1970).

${ }^{4}$ D. M. Larsen, Phys. Rev. B 2, 4209 (1970).

${ }^{5}$ G. E. Stillman, D. M. Larsen, C. M. Wolfe, and R. C. Brandt, Solid State Commun. 9, 2245 (1971).

${ }^{6}$ D. R. Cohn, D. M. Larsen, and B. Lax, Phys. Rev. B 6, 1367 (1972).

${ }^{7}$ R. S. Daries Bella and K. Navaneethakrishnan, Solid State Commun. 130, 773 (2004).

${ }^{8}$ S. T. Pérez-Merchancano, L. E. Bolivar-Marinez, and J. SilvaValencia, Rev. Mex. Fis. 53, 470 (2007).

${ }^{9}$ A. P. Wijnheijmer et al., Phys. Rev. Lett. 102, 166101 (2009).

${ }^{10}$ C. X. Xia, Z. P. Zeng, and S. Y. Wei, J. Appl. Phys. 106, 094301 (2009).
${ }^{11}$ L. D. Landau, Phys. Z. Sowjetunion 3, 664 (1933).

${ }^{12}$ S. I. Pekar, Zh. Eksp. Teor. Fiz. 19, 796 (1949).

${ }^{13}$ L. D. Landau and S. I. Pekar, Zh. Eksp. Teor. Fiz. 18, 419 (1948).

${ }^{14}$ G. D. Mahan, Many-Particle Physics (Plenum, New York, 1980), p. 502 .

${ }^{15}$ G. D. Mahan, Phys. Rev. 153, 983 (1967).

${ }^{16}$ G. D. Mahan and R. M. Mazo, Phys. Rev. 175, 1191 (1968).

${ }^{17}$ G. D. Mahan, Many-Particle Physics (Plenum, New York, 2000).

${ }^{18}$ Semiconductors, edited by O. Madelung, M. Schulz, and H. Weiss, Landolt-Börnstein, New Series III, Vols. 17a, 17b (Springer-Verlag, Berlin, 1982).

${ }^{19}$ G. D. Mahan and K. R. Subbaswamy, Local Density Theory of Polarizability (Plenum, New York, 1990).

${ }^{20}$ G. D. Mahan, Condensed Matter in a Nutshell (Princeton, New York, 2010), App. A2. 